\title{
¿QUÉ OCURRE CON LA ADAPTACIÓN Y EL RENDIMIENTO ACADÉMICO DE LOS ALUMNOS, EN UN CONTEXTO EDUCATIVO PLURICULTURAL?
}

Mª Inmaculada Ramírez, Francisco Herrera e Inmaculada Herrera

Universidad de Granada

\section{INTRODUCCIÓN Y REVISIÓN TEÓRICA}

Por desgracia, cada vez con mayor frecuencia, en nuestros centros escolares no es difícil observar a diario comportamientos discentes que hasta los menos expertos no dudarían en calificar como desadaptados. Por un sinfín de razones, nuestros alumnos parecen tener progresivamente mayores dificultades para su adaptación a este mundo tan complejo, donde los cambios se producen continuamente a velocidad de vértigo.

Y si, como la evidencia nos muestra, esto es así en los centros escolares que pudiéramos calificar de "normales", más o menos homogéneos y equilibrados en sus características constitutivas básicas fundamentales, qué ocurre, como es nuestro caso, en centros donde la pluriculturalidad es la nota singular común. Ése es nuestro interés; aunque, como no podía ser de otra manera, comenzaremos por realizar una breve revisión teórica al respecto.

La interpretación del continuum adaptación-inadaptación y su definición conceptual depende del enfoque hacia el que se quiera orientar: biológico, físico, psíquico y social; bien individualmente o en su conjunto.

Para tener una idea desde la perspectiva psicológica, que es la que aquí nos interesa, partimos del acuerdo con Hernández y Jiménez (1983, p. 27) en considerar la adaptación como un criterio operativo y funcional de la personalidad, en el sentido de que recoge la idea de ver hasta que punto los individuos logran estar satisfechos consigo mismos y si sus comportamientos son adecuados a los requerimientos de las distintas circunstancias en que tienen que vivir.

Un individuo puede tener una serie de características (personalidad) y puede estar más o menos ajustado (adaptación), constituyendo la propia adaptación una característica de la personalidad y siendo la personalidad la determinante de la adaptación. Ello significa que desde los rasgos de personalidad se puede predecir la adaptación y que desde ésta se puede definir la personalidad. Sin embargo, para efectos educativos y de intervención psicológica, nosotros preferimos manejar el concepto de adaptación, aunque dentro de ella existen diferentes rasgos de personalidad. Por otro lado, es preciso tener en cuenta a Quintana (1980, p. 14) cuando opina que la primera de las principales funciones sociales de la educación es la adaptación del individuo a las exigencias del grupo social. El individuo nace asocial («asocial» psicológicamente, aunque "social» antropológicamente; es decir, por el destino que le marca su naturaleza). Vivir es estar integrado en la sociedad; esta integración tiene sus exigencias: el individuo ha de aprenderlas, debe formarse los hábitos por ellas requeridos, y esos aprendizajes es la educación quien se los da. A esto lo llamamos socialización del individuo, que es un cometido, fundamentalmente, de la educación. Gracias a ella la sociedad incorpora a sí misma a sus nuevos miembros, perpetuando de este modo su existencia en el tiempo.

Cabe comparar esta función a la de la asimilación realizada por los seres vivos. Para algún autor extremista, como Spencer, no se trataría de una mera analogía, sino de una identidad real de funciones. La 
socialización del individuo es obra primordial de la educación familiar, hasta el punto de que Adorno llama a los padres «agentes de la sociedad».

Según Moraleda (1987, p. 436), el proceso de socialización se produce de dos posibles formas, a través de:

1ํ. La iniciación a la vida social por impregnación. La familia, su ambiente y su clima es un medio especialmente rico y complejo de relaciones interpersonales que favorece y multiplica...

$2^{2}$. La iniciación a la vida social por el control de la conducta. El desarrollo social significa la adquisición de la capacidad para comportarse de conformidad con las expectativas sociales...

Asimismo, siguiendo a Salmerón y Soriano (1989:187-200), el análisis del concepto de adaptaciónsupone la adopción de una serie de criterios que determinen lo que es considerado como "normal" y que, por tanto, definan las conductas en cuanto se ajusten o no a esa supuesta normalidad.

Desde una perspectiva tradicional en el análisis de la adaptación, el concepto de normalidad se basa generalmente en dos criterios:

$1^{\circ}$. Por una parte, en un criterio estadístico cuantitativo, según el cual se define como "normal" aquel comportamiento que reúne las características que determina la conducta de la mayor parte de un grupo social; por tanto, anormal es todo comportamiento que se aparta de dicha conducta generalizada.

$2^{\circ}$. Por otra parte, en un criterio estadístico que implica a su vez un criterio valorativo; según este último se considera como normal todo comportamiento que sea aprobado por el grupo o que no llegue a merecer su rechazo (Sánchez Moro, 1982).

Desde el enfoque interaccionista, la inadaptación social surge a partir de una situación conflictiva generada de forma permanente entre el individuo y el medio que le rodea. Las relaciones entre personas y situaciones dependen de las condiciones del sistema social en que están inmersos y la conducta del individuo se convierte en una respuesta adaptativa al ambiente que le circunda. En este sentido, la inadaptación se produce como una respuesta alternativa y adaptativa del indivi duo ante situaciones frustrantes.

Las posibles respuestas adaptativas o resultantes de la interacción individuo-medio dan lugar a diversos tipos de comportamientos:

a) Conformismo previo, caracterizado por la aceptación implícita del individuo, de la imposibilidad de acceder a determinadas metas culturales. Este tipo de relación no supone una inadaptación social y rara vez el sujeto entrará en conflicto con las leyes o instituciones de control de la sociedad, el individuo acepta su rol.

b) Comportamiento antisocial objetivo, manifestado por el sujeto que, en virtud de su peculiar relación con el medio, no acepta (aunque tal vez nunca se plantee de forma consciente) la imposibilidad de acceder a las metas sociales y busca caminos alternativos para conseguirlos.

c) Comportamiento social retirado, puesto en práctica por aquellos individuos que aún sin aceptar el conformismo pasivo no llegan a desarrollar de forma inevitable un comportamiento antisocial. Su característica fundamental consiste en un determinado alejamiento de la sociedad. Esta figura podría estar representada de una forma bastante aproximada por el individuo que "pasa de todo" (Salmerón y Soriano, 1989:188). 
Hoy se asume que el individuo es un ser en relación y la inadaptación supone fundamentalmente un conflicto relacional, por lo tanto toda desviación ha de ser diagnosticada al menos en dos direcciones: el individuo y su entorno social.

- Hacia el individuo, evaluando las posibilidades de que dispone para desenvolverse activamente en su relación social, lo que implica sobre todo acciones preventivas y acciones recuperadoras.

- Hacia el entorno social, en el que se ha configurado la situación de inadaptación y donde han de evaluarse alteraciones estructurales que deben ser recogidas.

En suma, podemos entender la adaptación como proceso dinámico encargado de ajustar los organismos a su medio (encargado de regular los comportamientos en función de su entorno), a través de los conocidos mecanismos piagetianos de asimilación (personalización de las influencias externas) y acomodación (reorganización de las propias estructuras internas en base a la asimilación), con el objetivo de conseguir la homeostasis (armonía, sintonía, equilibrio...). Y que, en el caso del hombre, esta organización le corresponde a su personalidad, actuando en función de dos mecanismos: uno subjetivo, el conocimiento y valoración de sí mismo (expectativas propias) y, otro objetivo, el conocimiento y valoración que los demás hacen de él (expectativas ajenas).

Y, por otra parte, en general, podemos entender la inadaptación como fenómeno que aparece cuando alguien no responde a sus propias expectativas o a las que los demás requieren de él. Si tal situación se da en función de su autoconcepto, de su control emocional, etc., como persona individual, podríamos hablar de inadaptación personal; mientras que cuando se da en su mundo de relación dentro del ámbito familiar, entonces podríamos calificarla de inadaptación familiar; si se da en el ámbito escolar, la etiquetaríamos de inadaptación escolar; y si se da en el ámbito comunitario, excluidos los dos ámbitos anteriores, entonces suele hablarse de inadaptación social. No obstante, hay autores que prefieren denominar a los tres últimos fenómenos inadaptación social, estableciendo entre ellos, unas veces, un tipo de relación evolutiva y, otras, una especie de determinismo recíproco o de correlación. Al parecer, los principales factores por los que se podría llegar a la inadaptación parecen proceder de diferentes tipos de entorno: carencial o patológico (biofisiológico, cognitivo...), familiar, escolar (de aprendizaje), social, laboral, comunitario, etc.

Queda patente, pues, que cuando el comportamiento que emite el individuo (derivado de su personalidad) es, generalmente, adecuado a sus propósitos o a las expectativas de los demás, se puede hablar de adaptación. Por tanto, el criterio de inadaptación lo marca la desviación de su comportamiento en relación con las expectativas propias o con las expectativas dominantes en su ambiente.

Como se puede apreciar, esas expectativas son extraordinariamente relativas culturalmente y varían con las personas, con el espacio y con el tiempo. Un ejemplo de ello lo tenemos en la ya clásica investigación de Wicman (1928) para determinar los problemas de conducta más graves de los escolares, utilizando una lista de 50 palabras. En ella se destaca la discrepancia de criterio entre psicólogos y maestros. Mientras los primeros, de 10 problemas analizados, calificaban 8 de inadaptación escolar, los más importantes; los maestros, ponían mayor relieve en los problemas de inadaptación social (7 entre 10).

Más tarde, en 1952, Stouffer repitió la investigación y no encontró gran diferencia entre psicólogos y maestros. En cambio, en 1959, realizó la investigación con 500 padres y la lista resultante fue semejante a la de 
los maestros de hacía 30 años. Incluso, acentuaban la importancia de los problemas de inadaptación social sobre los de adaptación personal. La valoración cultural, aún siendo relativa, pone también en evidencia la existencia de dos grandes tipos de inadaptación. Esto, por otra parte, ha quedado constatado en las clasificaciones psiquiátricas y en algunos análisis factoriales.

Según Zazzo (1978, p. 43) la inadaptación escolar, enfocada desde la perspectiva del caso clínico y terapias, supone la visión «a posteriori» de un problema que debería abordarse preventivamente a nivel práctico y dialécticamente a nivel teórico. Es preciso afrontar el estudio de la adaptación frente al de la inadaptación, y del seguimiento total de una población infantil, en lugar de las contrastaciones de desviaciones aisladas, basándose metodológicamente en la observación directa de los niños en la escuela y la indirecta de los testimonios de padres y maestros. Resaltando en primer plano el poder de la situación escolar (permisiva en preescolar y restrictiva en primaria) como factor clave de la adaptación.

Quay y Quay (1965) determinaron, factorialmente, tres tipos de inadaptación en el comienzo de la adolescencia. Una, que podía denominarse inadaptación personal, identificable con los aspectos neuróticos y que recoge aspectos como incapacidad para divertirse, sentimientos de inferioridad, preocupación, timidez, aislamiento, inseguridad, apatía, ensueño, distracción, aturdimiento, etc. Otra, que podría denominarse inadaptación social, identificable con la sociopatía, abarcando aspectos como ser inquieto, reclamar atención, perturbar, hacer ruidos, falto de atención e interés, perezoso en la escuela, irresponsable, desobediente, hiperactivo, etc. Y, la tercera, que constituye una mezcla de ambos.

En su estudio sobre 20.000 niños de escuela primaria, Lewis (1951) señaló que el 4\% presentaba problemas de ajuste personal y social suficientemente graves para ser tratados clínicamente, en consideración del maestro. Estos porcentajes, sin embargo, varían según los estudios. Así, Clancy y Smitter (1953), en California, lo ponían en el $11 \%$. Pero su criterio, en este caso, se basaba en la atención que debería tomar en cuenta el profesor. Mensh et al.(1959), en Missouri, establecieron, a través de una escala de Ullman y en colaboración con los maestros, que el $20 \%$ presentaba ciertas dificultades, mientras que el $8 \%$ tenía o era propenso a problemas serios como para recibir asistencia clínica. Davie et al.(1972), en un estudio longitudinal de todos los niños nacidos (1960) en el Reino Unido en la primera semana de marzo de 1958, determinó, a través de una escala dirigida a los maestros las siguientes proporciones: $64 \%$ estables, $22 \%$ levemente inadaptados y el $14 \%$ severamente inadaptados.

Se podrían citar otros estudios como los de Burt (1963), Micner (1967), Rogers (1968), Hevier (1973), Rutter (1975), etc.; donde lo característico es la alta variación de porcentajes entre ellos.

Pero, es fácil deducir que la variación en los porcentajes se debe a los criterios tomados. Hasta tal punto llega la variación, que Murphy (1956) estudió a un grupo de niños "normales", desde que asistían a la escuela maternal, y, después de cierto seguimiento, llegó a la conclusión de que ninguno estaba completamente libre de conflicto o problema de desarrollo. Algunos necesitaban ayuda especial de los padres, otros, necesitaban cambiar de escuela, o bien, atención especial del maestro o, incluso, hasta consulta psiquiátrica.

Todo esto refuerza la idea de que, en lugar de hablar de adaptados e inadaptados, mejor es hablar del continuo adaptación-inadaptación. Sin embargo, considerando las distintas investigaciones se puede determinar como promedio que sirve de guía, un $25 \%$ de casos con ciertos síntomas de desajuste y un $10 \%$ de casos con desajustes severos. 
Más importante que la variación de porcentajes según los criterios de inadaptación, es la variación de porcentajes según la clase social, el sexo o la cultura.

Al comparar la proporción de escolares inadaptados, entre la clase social más alta y la más baja se observa un incremento en esta última cercano al 17\% (Vall, 1973).

En cuanto al sexo, los niños muestran un menor control social, mayor agresividad, una actitud menos favorable hacia el aprendizaje y menor satisfacción con el ambiente familiar en comparación con las niñas (Hernández, 1983).

Por lo que se refiere a las diferencias culturales dentro del ámbito social, se observan:

a) Diferencias notables en la valoración del comportamiento por parte de los profesores y por parte de las madres.

b) Una misma conducta puede tener distinta tolerancia según los grupos sociales, por ejemplo, la destrucción de objetos ajenos o la agresividad.

c) Una misma conducta puede tener distinta significación psicológica según las distintas clases sociales. Por ejemplo, la destructividad que manifiesta un niño puede ser entendida en familias de profesiones liberales como un indicador de problemas emocionales; mientras que, esta misma conducta, en familias de clase trabajadora, donde es cinco veces más frecuente, adquiere una significación irrelevante.

Mckin y Cowen (1987, pp. 370-383), con el propósito de analizar la relación de cinco perspectivas de la adaptación de los niños en edad escolar: profesor, padres, compañeros, escala de valores personales y observaciones del comportamiento, y el propósito secundario de examinar la relación entre adaptación y obtención de conocimientos, comparando contextos urbano-suburbial, hombre-mujer y niños enviados o no a programas de adaptación, encontraron una concordancia baja-moderada entre las cinco perspectivas de adaptación y, respecto a la relación entre la adaptación y obtención de conocimientos, salieron peor parados los niños de suburbios, las niñas y los niños con problemas mentales, respectivamente.

Muchos inadaptados llegan con sus problemas a las escuelas normalmente; pero, otros, también numerosos, se van forjando día a día, desgraciadamente, a lo largo del proceso educativo, especialmente, como producto de malas actuaciones profesionales.

En el primer sentido, el fracaso escolar es una consecuencia de la inadaptación. El niño escolarmente inadaptado presenta síntomas muy variados que van desde las pequeñas indisciplinas de la clase al absentismo escolar. El resultado es siempre el mismo: el retraso del niño inadaptado, manifestado por su retraso académico.

Esta inadaptación se manifiesta de las siguientes formas:

1. Trastorno y variaciones en el rendimiento.

2. Trastornos de la conducta y las relaciones sociales con sus compañeros.

3. Trastornos de la salud física o mental.

El alumno inadaptado vive su situación como una dificultad de relación con el ambiente, lo que se traduce en problemas de relaciones personales con los educadores y compañeros, y en el enrarecimiento del ambiente familiar. Al percatarse de estas relaciones, se genera en él una difusa ansiedad y temor ante las calificaciones, 
que le producirá un fuerte sentido de inseguridad. Esta situación reviste especial dificultad en determinados momentos coyunturales de cambio de situación en las que existe un equilibrio relativo, sobre todo en los cambios de ciclos y, especialmente, en los de etapas educativas (ingreso en la escuela, paso de Educación Infantil a Primaria, de esta a Secundaria, etc.).

Dentro de este contexto, es conveniente analizar, particularmente, la repercusión de las dificultades de aprendizaje (learning disabilities) en la adaptación, ya que un creciente conjunto de investigaciones muestran que estos niños manifiestan problemas significativos de conducta o déficit en su desenvoltura social (Bender y Smith, 1990) y son a menudo mal aceptados por sus semejantes que no tienen esas dificultades (Bryan, 1974, 1976; Sheare, 1978; Scranton y Ryckman, 1979; Gresham y Reschly, 1986; Sater y Frech, 1989; Bursuck, 1989; Stone y LaGreca, 1990; Vaughn, Hogan, Kouzekanani y Shapiro, 1990).

Sin embargo, los resultados no han sido totalmente consistentes, en particular respecto a diferencias en estamento social entre niños con y sin dificultades de aprendizaje. Varios investigadores han demostrado que niños con dificultades, en comparación con sus semejantes sin ellas, son menos aceptados pero no más rechazados (Coben y Zigmond, 1986; Sater y French, 1989); aunque otros investigadores han encontrado que los niños con dificultades no son menos aceptados, pero si son más aislados socialmente (Prillaman, 1981).

Por otra parte, varios investigadores (Perlmutter et al., 1983; French y Waas, 1985; Kistner y Gatlin, 1989; Sater y French, 1989) han notado que aunque los niños con dificultades, como grupo, son menos adaptados socialmente que niños sin ellas, muchos de ellos no tienen grandes dificultades en las relaciones con sus semejantes.

Otra importante faceta de la adaptación social, es la perspicacia de los niños sobre su aceptación social. Si los niños piensan que son socialmente competentes y aceptados, es muy importante para su adaptación personal (Asher et al., 1990). A pesar de su estatus sociométrico, los niños que se dan cuenta de estar sin amigos, sufrirán probablemente de soledad y de sentimientos de inquietud personal (ansiedad social). Además de ser importantes por sí mismos, parece que tales sentimientos van a influir probablemente en las acciones interpersonales y en las relaciones entre semejantes.

Otro factor que, frecuentemente, influye en los resultados de los estudios sobre adaptación social de los niños con dificultades, es el marco en el que se les enseña. En la mayoría de los estudios previos, los niños con dificultades han sido integrados entre niños sin ellas solamente durante una parte del día escolar. Es decir, han sido sacados de la clase regular para recibir instrucción en un marco por separado (un aula de recursos o de integración). Los niños con dificultades han mostrado mantener una más favorable autopercepción de la competencia escolar, asistiendo en un marco separado, como opuesto a uno integrado (Renick y Harter, 1989). No obstante, no está claro si la integración influye en las percepciones de la aceptación social de esos niños, o en otras facetas de su adaptación social. Es frecuente que la integración parcial disminuya la proximidad y familiaridad física entre compañeros de clase con y sin dificultades de aprendizaje, lo que tiene un impacto negativo en sus relaciones con sus semejantes.

Como último apunte, decir que en la investigación de Juvonen y Bear (1992) no se encontraron diferencias significativas en las proporciones de niños con y sin dificultades de aprendizaje entre los grupos sociométricos aceptados e impopulares. Dos tercios de niños tuvieron al menos un amigo recíproco y más de la mitad tuvo 
amistad con un compañero de clase sin dificultades de aprendizaje. Las niñas con dificultades de aprendizaje recibieron el número más alto de muestras negativas y fueron las menos preferidas. Además, comparaciones entre grupos de niños adaptados y no adaptados socialmente, con y sin dificultades de aprendizaje, mostraron que los maestros se dieron cuenta de que los niños con dificultades adaptados socialmente resultaron ser social y académicamente menos competentes que los niños adaptados sin dificultades. Estos resultados fueron discutidos teniendo en cuenta los efectos del marco educacional y de la composición de las clases, sobre la adaptación social de los niños.

En nuestro contexto pluricultural concreto es preciso tener en cuenta que el punto de vista de la adaptación a una nueva cultura es el producto de múltiples factores interactivos, lo cual ha dirigido el desarrollo de varios prototipos de adaptaciones multidimensionales (Kagan y Cohen, 1990). Básicamente, la mayoría de estos modelos presentan el proceso de aculturación como si requirieran alguna graduación de reestructuración cognitiva. Uno de los prototipos más tempranos fue introducido por Goldlust y Richmond en 1974, cuyo modelo incluía: 1. Internalización, 2. Identificación, 3. Cambio de valores y actitudes y 4. Influencias premigratorias.

Atkinson, Morten y Sue (1989) propusieron un modelo de cinco etapas en el desarrollo de la identidad que pretende explicar cómo responden los inmigrantes y/o las minorías a la cultura mayoritaria. Este modelo incluía: 1. Conformidad (individuos que no se gustan a sí mismo y admiran a los miembros de la cultura dominante), 2. Disonancia (conflictos que surgen entre los sentimientos de desprecio y aprecio individuales para con ellos mismos, punto de vista sostenido por la cultura mayoritaria y otros grupos minoritarios, y sus propios sentimientos personales), 3. Resistencia e inmersión (los individuos comienzan a apreciarse a ellos mismos, los sentimientos étnicos resurgen y empiezan a despreciar la cultura mayoritaria), 4. Introspección (los individuos exploran y examinan las razones para que se gusten ellos mismos o que les guste su grupo y las bases etnocéntricas para juzgar a otras minorías y a la cultura dominante), y 5 . Articulación sinergética y consciencia (los individuos comienzan a aceptar y respetar los valores de la cultura diferente de otros grupos minoritarios, de la cultura dominante, y de la suya propia).

Mendoza y Martínez (1981) presentaron otro modelo de aculturación que proponían los siguientes niveles: 1. Modalidades de dimensión que incluyen varios componentes de factores cognitivos, afectivos y de comportamiento; y 2. Tipos de dimensión consistentes en seis fases: asimilación cultural, extinción de la cultura nativa, resistencia cultural, cambio cultural, incorporación cultural y transmutación cultural;

Otros investigadores han reconocido factores adicionales que impactan en el proceso de aculturación tales como la influencia del lenguaje, religión, actividades sociales y vida familiar (Rosenthal y Hrynevich, 1985).

Además de la transición de la aculturación, existen otros factores que pueden impactar en el proceso de adaptación de los niños inmigrantes a las escuelas. Sewel-Coker et al. (1985) afirmaron que los inmigrantes valoran la propiedad (la propiedad es asociada con la seguridad económica, la libertad y el estatus alto). Como resultado, los padres suelen trabajar en más de una ocupación para adquirir propiedades y crear oportunidades para sus hijos. El hecho de llevar adelante múltiples trabajos afecta al control de los padres sobre los hijos en una época en la que más lo necesitan y resulta difícil para los padres realizar apoyos en los asuntos escolares o ayuda a sus hijos con el trabajo escolar (Thrasher y Anderson, 1988).

En el colegio, estos niños pueden ser discriminados por los compañeros y, en algunos casos, 
encasillados por sus acentos y por sus pobres conocimientos académicos. Esto representa una fuente de estrés que fomenta la baja autoestima, la pobre autoconfianza, ansiedad, sentimientos de inferioridad y soledad (Yao, 1985; Esquivel y Keitel, 1990). Alternativamente, estas condiciones emocionales afectan a los progresos académicos o al aprendizaje tanto como la adaptación social. Precisamente, Thrasher y Anderson (1988) revelaron en su estudio que el 30\% de su muestra estaban incluidos en Educación Especial. Claramente, es posible que estos niños estén desclasificados porque sus dificultades no son bien entendidas. Una gran parte de las dificultades que los niños inmigrantes han experimentado son relativas a la incapacidad de nuestras escuelas para dirigir sus necesidades, lo que se ha visto agravado enormemente en aquellos casos de procedencia de zonas en guerra o de hambruna (afortunadamente, no es nuestro caso).

La adaptación a la escuela exige que el niño, especialmente el inmigrante o de minorías, aprenda rápidamente cómo funciona todo en ese entorno, para garantizarse el máximo de recompensas y el mínimo de penalizaciones, para exhibir las evaluaciones positivas y ocultar las negativas, y para conseguir la aprobación simultánea de dos audiencias (profesor y compañeros), lo cual no siempre es fácil.

En vista de todo lo expuesto, es preciso tomar en consideración que los padres y profesores de inmigrantes o de minorías necesitan guías para ayudar a sus hijos y alumnos aculturados, de manera que les faciliten la posibilidad de triunfar en las escuelas, así como los servicios escolares necesarios para llegar a sensibilizarse y concienciarse sobre las dificultades experimentadas por ellos.

Es esencial llegar a familiarizarse con la historia, la cultura y los factores que precipitaron la migración de las familias inmigrantes que puedan encontrarse en el distrito escolar local, para sentirse a gusto trabajando con ellos y sus familias, hay que acostumbrarse a su lenguaje, a sus prácticas religiosas, a sus expectativas escolares y comportamientos, a sus expectativas en el papel del sexo y del trabajo étnico, y hasta visitar sus comunidades y casas, comer su comida y vivenciar diferentes aspectos de su etnia, cultura, religión, etc.; en suma, lo que llamamos Educación no sólo Intercultural; sino, además, Intracultural (Ramírez Salguero, 1997).

\section{EL PROBLEMA}

La motivación, necesidad e importancia socioeducativa de esta investigación pudiera radicar en que a nivel intuitivo-experiencial, como podría asegurar cualquier docente de la Ciudad Autónoma de Ceuta (España), nos encontramos ante una situación de desigualdad escolar y, por tanto, social, que frena el desarrollo de un grupo social sumergido en otro dominante y que, lejos de ofrecer medios para paliarla, no hace más que acrecentar sus diferencias, en el mejor de los casos, por ignorancia voluntaria de lo que es una evidencia, con lo que se confirma que no hay mayor ciego que el que no quiere ver...

Precisamente por ello, es necesario analizar adecuadamente la situación actual y sus antecedentes, como punto de partida, para poder dimensionarla y, de esta forma, iniciar la puesta en marcha de medidas que contribuyan a minimizar o eliminar (cuestión utópica) las desigualdades, lo que sin duda alguna contribuirá al mejor funcionamiento y desarrollo social (pacífico, democrático, etc.); de ahí, su importancia actual y futura.

El problema fundamental con el que nos encontramos es que la población escolar de la Comunidad Musulmana Ceutí sufre un alto déficit en su rendimiento académico, constatado especialmente por la evidencia de los datos aportados por las evaluaciones académicas (profesores/MEC), lo que demuestra un alto índice de fracaso escolar. Dado que se trata de una minoría étnica con cantidad de carencias, sobre todo, socio-económicas 
y educativo-culturales; tal vez, su posible mala adaptación tenga algo que ver con todos los problemas que padecen.

Se ha planteado el siguiente problema general: ¿Qué efectos provocan los diferentes tipos y niveles de adaptación de la población escolar de las Comunidades Ceutíes Cristiana y Musulmana en su rendimiento académico, especialmente en esta última?.

Evidentemente, existen indicios experienciales que hacen pensar que esta población está mal adaptada y que ello influye negativamente en su rendimiento académico, como confirmaría cualquier persona relacionada con el mundo educativo ceutí; no obstante, conviene conocer con rigor ¿en qué medida?, ¿cómo y por qué ocurre? y, lo que sería mucho más importante, ¿qué se hace o qué hacer para paliarlo?.

Este problema general, para su mejor estudio y análisis, hemos decidido descomponerlo en los siguientes problemas específicos:

1. ¿Qué tipos y niveles de adaptación tiene la población escolar de las comunidades ceutíes cristiana y musulmana, individualizada y diferencialmente, por culturas y sexos?.

2. ¿Qué tipos y niveles de rendimiento académico tiene la población escolar de las comunidades ceutíes cristiana y musulmana, individualizada y diferencialmente, por culturas y sexos?

3. ¿Qué relaciones existen entre la adaptación y el rendimiento académico de los alumnos de la población escolar de las comunidades ceutíes cristiana y musulmana, individualizada y diferencialmente, por culturas y sexos?

\section{HIPÓTESIS}

En consecuencia, se han formulado las siguientes hipótesis:

I. General: La adaptación de la población escolar de las comunidades ceutíes cristiana y musulmana, afecta significativamente a su rendimiento académico, especialmente a la última.

II. Específicas:

1. Existen diferencias significativas en los tipos y niveles de adaptación de la población escolar de las comunidades ceutíes cristiana y musulmana, individualizada y diferencialmente, por cutturas y sexos.

2. Existen diferencias significativas en los tipos y niveles de rendimiento académico de la población escolar de las comunidades ceutíes cristiana y musulmana, individualizada y diferencialmente, por culturas y sexos.

3. Existen relaciones significativas entre la adaptación y el rendimiento académico de los alumnos de la población escolar de las comunidades ceutíes cristiana y musulmana, individualizada y diferencialmente, por culturas y sexos.

\section{MÉTODO}

\subsection{Muestra}

La muestra fue extraída de la población escolar de Ceuta de Educación Primaria (segundo y tercer ciclo) y de E.S.O. (Primer ciclo) por muestreo estratificado con afijación proporcional (según el nivel y la etapa educativa, el grupo cultural -cristiano y musulmán-, y la cultura dominante en el centro educativo); garantizando un nivel de 
confianza del $95,5 \%$ y un margen de error del $3 \%$.

Del total de los sujetos que componen la muestra (1315), el $49,8 \%$ son chicos y el resto chicas, perteneciendo el $50,8 \%$ de ellos a la comunidad cristiana y el resto a la musulmana.

\subsection{Instrumentos y variables}

El instrumento de evaluación de la adaptación empleado ha sido el Inventario de Adaptación de Conducta (IAC), de De la Cruz y Cordero (1981). Es un inventario integrado por 123 frases, a las que el sujeto debe responder de acuerdo con su manera de pensar y actuar. Su fiabilidad oscila desde 0,81 (para la dimensión de adaptación personal) a 0,97 (para la adaptación global).

Con este inventario se evalúan las variables siguientes: Adaptación personal, familiar, escolar, social y global.

\subsection{Resultados}

4.3.1. Diferencias en los tipos y niveles de adaptación de la población escolar de las comunidades ceutíes cristiana y musulmana, individualizada y diferencialmente, por culturas y sexos

En este apartado damos cuenta de los resultados obtenidos en los tipos y niveles de adaptación de la población escolar de las comunidades ceutíes cristiana y musulmana, individualizada y diferencialmente, por culturas y sexos.

En la tabla 1 se exponen los resultados de la prueba $t$ para el contraste de medias cuando la variable de agrupamiento es el tipo de grupo (cristianos-musulmanes) y la variable agrupada es la puntuación obtenida en los diferentes factores de adaptación que se evalúan con el IAC.

En esta tabla se puede observar que los valores de $t$ asociados a las diferencias de medias en adaptación personal, familiar, escolar y global son estadísticamente significativos $(p<0,05)$, no superando este criterio la adaptación social.

Así pues, podemos decir que el alumnado cristiano está mejor adaptado que el musulmán, salvo en adaptación social, donde no se aprecian diferencias significativas entre ambos grupos.

En la tabla 2 se exponen los resultados de la prueba $t$ para el contraste de medias cuando la variable de agrupamiento es el sexo (chicos-chicas), del total del alumnado de la muestra, y la variable agrupada es la puntuación obtenida en los diferentes factores de adaptación que se evalúan con el IAC.

En esta tabla se puede observar que los valores de $t$ asociados a las diferencias de medias en adaptación familiar, escolar y global son estadísticamente significativos $(p<0,05)$, no superando este criterio el resto.

Por lo tanto, en general (para todos los cristianos y musulmanes juntos), podemos decir que las alumnas están mejor adaptadas a la escuela que los alumnos.

Por otra parte, manifestar que en cuanto a las diferencias por sexo, dentro del alumnado cristiano, se han hallado diferencias estadísticamente significativas en adaptación escolar entre chicos y chicas, a favor de estas últimas $(p<0,05)$; mientras que, dentro del alumnado musulmán, no se han encontrado diferencias estadísticamente significativas entre chicos y chicas. 
4.3.2. Diferencias en los tipos y niveles de rendimiento académico de la población escolar de las comunidades ceutíes cristiana y musulmana, individualizada y diferencialmente, por culturas y sexos

En este apartado damos cuenta de los resultados obtenidos en los tipos y niveles de rendimiento académico de la población escolar de las comunidades ceutíes cristiana y musulmana, individualizada y diferencialmente, por culturas y sexos.

En la tabla 3 se exponen los resultados de la prueba $t$ para el contraste de medias cuando la variable de agrupamiento es el tipo de grupo (cristianos-musulmanes) y la variable agrupada es la puntuación obtenida en rendimiento académico.

En esta tabla se puede observar que todos los valores de $t$ asociados a las diferencias de medias en rendimiento académico son estadísticamente significativos $(p<0,05)$.

Así pues, podemos decir que el alumnado cristiano tiene mejor rendimiento académico que el musulmán, tanto por áreas curriculares como globalmente.

Asimismo, destacar que, en general (para todo el alumnado, cristiano y musulmán juntos), en función del sexo, no se aprecian diferencias estadísticamente significativas.

Por otra parte, manifestar que en cuanto a las diferencias por sexo, dentro del alumnado cristiano, no se han hallado diferencias estadísticamente significativas entre los chicos y las chicas en su rendimiento académico; mientras que, dentro del alumnado musulmán, tan sólo se han encontrado diferencias estadísticamente significativas en el caso del rendimiento en el Área de Ciencias Sociales, a favor de las chicas $(p \quad 0,000)$.

4.3.3. Relaciones existentes entre la adaptación y el rendimiento académico de los alumnos de la población escolar de las comunidades ceutíes cristiana y musulmana, individualizada y diferencialmente, por culturas y sexos

Tomando los resultados que se resumen en la tabla 4 , se puede observar que en la comunidad escolar ceutí, cristiana y musulmana conjuntamente, el rendimiento académico global del alumnado está determinado por las variables siguientes: adaptación escolar, familiar y personal. El conjunto de todas ellas da cuenta del 71,17\% de la varianza en el rendimiento académico $\left(R^{2}=0,7117\right)$, si bien el mayor poder determinante lo ejerce el factor de adaptación escolar, que por sí sola explica el $40,90 \%$ de la varianza en rendimiento académico.

La función descrita sería la siguiente:

\section{Rendimiento Académico Global $=0,4529$ a. escolar $+0,3122$ a. familiar $+0,2798$ a. personal $-0,1240$}

Como se puede apreciar, existe una relación directamente proporcional entre la adaptación y rendimiento académico de la población escolar de las comunidades ceutíes cristiana y musulmana, individualizada y diferencialmente, por culturas y sexos, que en el caso del alumnado musulmán se manifiesta de forma más clara entre su baja adaptación y su bajo rendimiento académico.

El análisis se ha llevado a cabo empleando el método de regresión múltiple paso a paso o "stepwise", considerando como variable criterio el rendimiento académico y como variables predictoras todas las demás.

Para su interpretación debemos fijarnos en el último paso y, dentro de él, en el orden de entrada de las variables en la ecuación de regresión, en el valor de â de cada variable, en su significación, y, a nivel más general, 
en el índice de regresión (R) y en el coeficiente de determinación múltiple $\left(R^{2}\right)$, que indica el porcentaje de varianza explicada por el conjunto de las variables predictoras incluidas en la ecuación.

Por otra parte, para los alumnos de la comunidad ceutí cristiana la única variable que predice su rendimiento académico global es la adaptación escolar, que explica el 31,61 \% (â $=0,3161, t=14,13$ y p =0,000).

Y, para los alumnos de la comunidad ceutí musulmana, también la única variable que predice su rendimiento académico global es la adaptación escolar, que explica el $47,21 \%$ (â $=0,4721, \mathrm{t}=10,74$ y $\mathrm{p}=$ $0,000)$.

\section{CONCLUSIONES Y DISCUSIÓN}

Tras el análisis de los datos obtenidos podemos concluir lo siguiente:

\subsection{Respecto a la hipótesis general}

Podemos afirmarla en el sentido que indican las hipótesis específicas (subhipótesis) planteadas: La adaptación de la población escolar de las comunidades ceutíes cristiana y musulmana, afecta significativamentea su rendimiento académico, especialmente a la última.

\subsection{Respecto a la primera hipótesis específica}

Podemos afirmarla en el siguiente sentido:

1. En el caso de la población escolar de la comunidad ceutí, por culturas: cristiana y musulmana.

Existen diferencias estadísticamente significativas en todos los factores de la adaptación analizados excepto en adaptación social, obteniendo los mejores resultados los alumnos cristianos; es decir, éstos son los que parecen estar mejor adaptados.

2. En el caso de la población escolar de la comunidad ceutí, por sexos: chicos y chicas.

a) En general, para todos los alumnos cristianos y musulmanes juntos, podemos decir que existen diferencias estadísticamente significativas en la adaptación familiar, escolar y global, obteniendo las chicas los mejores resultados.

b) En el alumnado cristiano, se han hallado diferencias estadísticamente significativas en la adaptación escolar entre chicos y chicas, obteniendo las chicas los mejores resultados.

c) En el alumnado musulmán, no se han encontrado diferencias estadísticamente significativas entre chicos y chicas.

\subsection{Respecto a la segunda hipótesis específica}

Podemos afirmarla en el siguiente sentido:

1. En el caso de la población escolar de la comunidad ceutí, por culturas: cristiana y musulmana.

Existen diferencias estadísticamente significativas en el rendimiento académico en todas las áreas curriculares y globalmente, obteniendo los mejores resultados los alumnos cristianos.

2. En el caso de la población escolar de la comunidad ceutí, por sexos: chicos y chicas.

a) En general, para todo el alumnado cristiano y musulmán junto, en cuanto a las diferencias en el rendimiento académico por sexo, podemos decir que no existen diferencias estadísticamente significativas entre chicos y chicas.

b) En el alumnado cristiano, no se han hallado diferencias estadísticamente significativas entre los chicos y las chicas en su rendimiento académico. 
c) En el alumnado musulmán, tan sólo se han encontrado diferencias estadísticamente significativas en el caso del rendimiento en el Área de Ciencias Sociales, siendo las chicas las que obtienen mejores resultados.

\subsection{Respecto a la tercera hipótesis específica}

Podemos afirmarla en el siguiente sentido:

1. En el caso de la población escolar de la comunidad ceutí, por culturas: cristiana y musulmana.

En la comunidad escolar ceutí, cristiana y musulmana conjuntamente, el rendimiento académico global del alumnado está determinado por las variables siguientes: adaptación escolar, adaptación familiar y adaptación personal. Es decir, estos factores de la adaptación, predicen el rendimiento académico de los alumnos.

2. En el caso de la población escolar de la comunidad ceutí cristiana.

Para la comunidad ceutí cristiana la única variable que predice el rendimiento académico global es la adaptación escolar.

3. En el caso de la población escolar de la comunidad ceutí musulmana.

También, para la comunidad ceutí musulmana la única variable que predice el rendimiento académico global es la adaptación escolar.

Finalmente manifestar que, aunque no conocemos trabajos realizados en una línea y contexto similares, podríamos decir que es congruente con los trabajos realizados por Vall (1973) al comparar la proporción de escolares inadaptados, observando que entre la clase social más alta y la más baja se produce un incremento en esta última cercano al $17 \%$, en el sentido de interpretar como clase social más alta a la mayoría autóctona dominante (cristiana) y como clase más baja a la minoría inmigrada (musulmana). En cuanto al sexo, se aprecian diferencias estadísticamente significativas entre chicos y chicas en su adaptación escolar que, quizás, pudiera explicar la afirmación de Hernández (1983) en el sentido de que los niños muestran menor control social, mayor agresividad, una actitud menos favorable hacia el aprendizaje y menor satisfacción con el ambiente familiar en comparación con las niñas.

\section{BIBLIOGRAFÍA}

ASHER, S.R. et al., Peer rejection and loneliness in childhood. In Asher, S.R. y Coie, J.D.: Peer rejetion in childhood. Cambridge. Cambridge, University Press., 1990.

ATKINSON, D.R.; MORTEN, G. \& SUE, D.W. , Counseling american minorities. Wisconsin, Brown, 1989.

BENDER, W.N. \& SMITH, J.K., Classroom behavior in children and adolescents with learning disabilities: a metaanalysis. Journal of Learning Disabilities, 23, 1990, 265-273.

BRYAN, T.J., Peer popularity of learning disabled children. Journal of Learning Disabilities, 1, 1974, 59-67.

BRYAN, T.J., Peer popularity of learning disabled children: A Replication. Journal of Learning Disabilities, 9, 1976, 163-175.

BURSUCK, W.D., A comparison of student with learning disabilities to low achieving and higher achieving student on three dimensions of social competence. Journal of Learning Disabilities, 22, 1989, 227-235.

CLANCY, N. \& SMITTER F., A study of emotionally disturbed children in Santa Bárbara Country Schools. California Journal Education, 4, 1953, 84-93.

COBEN, S.S. \& ZIGMOND, N., The social integration of learning disabled student from self-contained to mainstream elementary settings. Journal of Learning Disabilities, 19, 1986, 154-163. 
DAVIE, R. et al., From birth to seven. London: Longman, 1972.

DE LA CRUZ, M.V. y CORDERO, A., Inventario de Adaptación de Conducta (IAC). Madrid: TEA, 1981.

ESQUIVEL, G. \& KEITEL, M., Counseling immigrant children in the schools. Elementary School Guidance and Counseling, 24, 1990, 211-219.

FRENCH, D.C. \& WAAS, G.A., Teachers ability to identify peer-rejected children: a comparison of sociometrics and teacher ratings. Journal of School Psychology, 23, 1985, 182-190.

GOLDLUST, J. Y RICHMOND, A.H., A multivariate model of immigrant adaptation. International Migration, 8, 1974, 91-100.

GRESHAM, F.M. \& RESCHLY, D.J., Social skills deficits and low peeracceptance of mainstreamed learning disabled children. Learning Disability Quartely, 9, 1986, 169-177.

HERNÁNDEZ, P., Rendimiento, adaptación e intervención psicoeducativa. Tenerife: Secretariado de Publicaciones de la Universidad de La Laguna, 1983.

HERNÁNDEZ, P. Y JIMÉNEZ, J.E., Intervención psicoeducativa y adaptación. Una alternativa de salud mental desde la escuela. Tenerife: Secretariado de Publicaciones de la Universidad de La Laguna, 1983.

JUVONEN, J. \& BEAR, G., Social adjustment of children with and without learning disabilities in integrated classrooms. Journal of Education Psychology, 84 (2), 1992, 30-42.

KAGAN, H. \& COHEN, J., Cultural adjustment of international students. Psychological Sciences, 1, 1990, 117-125.

KISTNER, J.A. \& GATLIN, D., Correlates of peer rejection among children with learning disabilities. Learning Disability Quartely, 12, 1989, 86-93.

LEWIS, H.H., A country guidance department looks at its problem behavior children. California Journal Education, 1, 1951, 29-38.

McKIM, K.S. \& COWEN, E.L., Multiperspective assesment of young childre'n school adjustment. School Psychology Review, 16 (3) 1987, 370-383.

MENDOZA, R.H. \& MARTíNEZ, J.L., The measurement of acculturation. En Baron, J.: Exploration in Chicago Psychology. New York: Praeger, 1981.

MENSH, I.N., Children's behavior symptoms and their relationship to school adjustment, sex, and social class. Journal Social Issues, 15 (1), 1959, 135-43.

MORALEDA, M., Privación cultural, dificultades verbales y fracaso escolar. Bordón, 267, 1987, 185-198.

MURPHY, L.B., Personality in young children. Vol.I. Methods for the study of personality in young children. New York: Basic Books, 1956.

PERLMUTTER, J., Sociometric status and related personality characteristics of mainstreamed learning disabled adolescents. Learning Disability Quartely, 6, 1983, 76-85.

Prillaman, D., Acceptance of learning disabled student in the mainstream enviroment: a failure to replicate. Journal of Learning Disabilities, 14, 1981, 123-131.

QUAY, H.C. \& QUAY, L.C., Behavior problems in early adolescence. Child Development, 36, 1965, 247-255.

QUINTANA, J.M., Sociología de la educación: La enseñanza como sistema social. Barcelona: Hispano Europea, 1980.

RAMÍREZ, M.I., La adaptación como factor de rendimiento de la población escolar de la comunidad musulmana ceutí. Ceuta: UNED, 1997. 
RENICK, M.J. \& HARTER, S., Impact of social comparisons on the development self-perceptions of learning disabled student. Journal of Educational Psychology, 81, 1989, 93-102.

ROSENTHAL, P.A. \& HRYNEVICH, C., Ethnicity and ethnic identity: a comparative study of greek-italian and anglo-australian adolescents. International Journal of Psychology, 20, 1985, 319-327.

SALMERÓN, H. y SORIANO, A., Diagnóstico de la inadaptación social en el período de la adolescencia. Revista de Educación de la Universidad de Granada, 3, 1989, 187-200.

SATER, F.M. \& FRENCH, D.C., A comparison of the social competencies of learning disabled and low achieving elementary children. Journal of Special Education, 23, 1989, 111-119.

SCRANTON, T.R. \& RYCKMAN, D.B., Sociometric status of learning disabled children in an integrative program. Journal of Learning Disabilities, 12, 1979, 223-231.

SHEARE, J.B., The impact of resource programs upon the self-concept and peer acceptance of learning disabled children. Psychology in the Schools, 15, 1978, 63-71.

SEWEL-COKER, B.; HAMILTON-COLLINS, J. \& FEIN, E., Social work practice with west indian immigrants. Social casework. The Journal of Contemporary Social Work, 11, 1985, 351-357.

STONE, W.L. \& LAGRECA, A.M., The social status of children with learning disabilities: a reexamination. Journal of Learning Disabilities, 23, 1990, 58-66.

STOUFFER, G.A.W., Behavior problems of children as viewed by teachers and mental hygienists. Ment Hyg, 36, 1952, 77-85.

STOUFFER, G.A.W., The attitudes of parents toward certain behavior problems of children, research for the teaching profession. Indiana, Pa: State Teachers. College, 1959.

THRASHER, S. \& ANDERSON, G., The west indian family: treatment challenges. Social Casework. The Journal of Contemporary Social Work, 17, 1988, 129-136.

VALL, W.D., The problem child schools. London Educational, 2, 1973, 91-99.

VAUGHN, S.; HOGAN, F.; KOUZEKANANI, H. \& SHAPIRO, J., Peer acceptance, self-perceptions and social skills of learning disabled student prior to identification. Journal of Educational Psychology, 82, 1990, 285293.

WICMAN, E.K., Childrens's behaviour and teacher's attitudes. New York: Commonwealth Fund, 1928.

YAO, E.L., Adjustment needs of asian immigrant children in the schools. Elementary School Guidance and Counseling, 19, 1985, 167-174.

Zazzo, B., La adaptación escolar, un estudio experimental. Infancia y Aprendizaje, 41, 1978, 112-125.

\section{Tabla 1}

Prueba $t$ de Student para el contraste de medias de muestras independientes, considerando como variable de agrupamiento el tipo de grupo: cristianos y musulmanes, y como variable agrupada las puntuaciones en los distintos factores de la adaptación.

\begin{tabular}{|l|l|c|c|c|c|c|c|}
\hline \multicolumn{2}{|c|}{ Variables } & \multicolumn{3}{c|}{ Estadísticos } & \multicolumn{3}{c|}{ Prueba $t$} \\
\hline Adaptación & Grupos & $\mathrm{n}$ & $\mathrm{x},-$ & $\mathrm{o}$ & $\mathrm{t}$ & g.l. & $\mathrm{p}$ \\
\hline & & & & & & & \\
\hline
\end{tabular}




\begin{tabular}{|c|c|c|c|c|c|c|c|}
\hline Personal & $\begin{array}{c}\text { Cristianos } \\
\text { Musulmanes }\end{array}$ & $\begin{array}{c}668 \\
647\end{array}$ & $\begin{array}{c}49,87 \\
39,65\end{array}$ & $\begin{array}{c}21,89 \\
22,73\end{array}$ & 0,31 & 1313 & $\mathbf{0 , 0 0 0}$ \\
\hline \multirow{2}{*}{ Familiar } & Cristianos & 668 & 55,29 & 24,18 & 14,3 & 1313 & $\mathbf{0 , 0 0 0}$ \\
& Musulmanes & 647 & 37,06 & 21,95 & & & \\
\hline \multirow{2}{*}{ Escolar } & Cristianos & 668 & 51,24 & 24,35 & 6,74 & 1313 & $\mathbf{0 , 0 0 0}$ \\
& Musulmanes & 647 & 42,60 & 22,06 & & & \\
\hline \multirow{2}{*}{ Social } & Cristianos & 668 & 70,73 & 22,72 & 1,80 & 1313 & 0,072 \\
& Musulmanes & 647 & 68,39 & 24,50 & & & \\
\hline \multirow{2}{*}{ Global } & Cristianos & 668 & 227,4 & 72,55 & 9,86 & 1313 & $\mathbf{0 , 0 0 0}$ \\
& Musulmanes & 647 & 187,7 & 73,46 & & & \\
\hline
\end{tabular}

Tabla 2

Prueba $t$ de Student para el contraste de medias de muestras independientes, considerando como variable de agrupamiento el sexo: chicos y chicas, del total del alumnado, y como variable agrupada las puntuaciones en los distintos factores de la adaptación.

\begin{tabular}{|c|c|c|c|c|c|c|c|}
\hline \multicolumn{2}{|c|}{ Variables } & \multicolumn{3}{c|}{ Estadísticos } & \multicolumn{3}{c|}{ Prueba $t$} \\
\hline \multirow{2}{*}{ Adaptación } & Grupos & $\mathbf{n}$ & $\mathbf{x},-$ & $\mathbf{o}$ & $\mathbf{t}$ & $\mathbf{g} . \mathbf{l}$. & $\mathbf{p}$ \\
\hline \multirow{2}{*}{ Personal } & Chicos & 655 & 44,20 & 22,82 & $-1,00$ & 1313 & 0,310 \\
& Chicas & 660 & 45,48 & 22,94 & & & \\
\hline \multirow{2}{*}{ Familiar } & Chicos & 655 & 44,94 & 24,62 & $-2,00$ & 1313 & $\mathbf{0 , 0 0 0}$ \\
& Chicas & 660 & 47,69 & 24,99 & & & \\
\hline \multirow{2}{*}{ Escolar } & Chicos & 655 & 44,32 & 24,02 & $-4,10$ & 1313 & $\mathbf{0 , 0 0 0}$ \\
& Chicas & 660 & 49,64 & 22,99 & & & \\
\hline \multirow{2}{*}{ Social } & Chicos & 655 & 69,96 & 24,10 & 0,58 & 1313 & 0,560 \\
& Chicas & 660 & 69,20 & 23,18 & & & \\
\hline \multirow{2}{*}{ Global } & Chicos & 655 & 203,5 & 73,44 & $-2,10$ & 1313 & $\mathbf{0 , 0 0 0}$ \\
& Chicas & 660 & 212,2 & 77,55 & & & \\
\hline
\end{tabular}

Tabla 3

Prueba $t$ de Student para el contraste de medias de muestras independientes, considerando como variable de agrupamiento el tipo de grupo: cristianos y musulmanes, y como variable agrupada las puntuaciones en rendimiento académico.

\begin{tabular}{|c|c|c|c|c|c|c|c|}
\hline \multicolumn{2}{|c|}{ Variables } & \multicolumn{3}{c|}{ Estadísticos } & \multicolumn{3}{c|}{ Prueba $t$} \\
\hline & Grupos & $\mathrm{n}$ & $\mathrm{x},-$ & $\mathrm{o}$ & $\mathrm{t}$ & g.l. & $\mathrm{p}$ \\
\hline
\end{tabular}




\begin{tabular}{|c|c|c|c|c|c|c|c|}
\hline Lengua & $\begin{array}{c}\text { Cristianos } \\
\text { Musulmanes }\end{array}$ & $\begin{array}{c}668 \\
647\end{array}$ & $\begin{array}{c}5,99 \\
4,80\end{array}$ & $\begin{array}{c}1,49 \\
1,47\end{array}$ & 14,6 & 1313 & $\mathbf{0 , 0 0 0}$ \\
\hline \multirow{2}{*}{ Matemáticas } & Cristianos & 668 & 5,80 & 1,62 & 5,70 & 1313 & $\mathbf{0 , 0 0 0}$ \\
& Musulmanes & 647 & 5,33 & 1,38 & & \\
\hline \multirow{2}{*}{ CC. Naturales } & Cristianos & 668 & 6,03 & 1,36 & 7,60 & 1313 & $\mathbf{0 , 0 0 0}$ \\
& Musulmanes & 647 & 5,47 & 1,30 & & & \\
\hline \multirow{2}{*}{ CC. Sociales } & Cristianos & 668 & 5,81 & 1,51 & 8,80 & 1313 & $\mathbf{0 , 0 0 0}$ \\
& Musulmanes & 647 & 5,06 & 1,56 & & & \\
\hline \multirow{2}{*}{ Global } & Cristianos & 668 & 5,69 & 1,70 & 9,90 & 1313 & $\mathbf{0 , 0 0 0}$ \\
& Musulmanes & 647 & 4,82 & 1,49 & & & \\
\hline
\end{tabular}

Tabla 4

Regresión múltiple paso a paso "stepwise", considerando como variable criterio el rendimiento académico y como variable predictora la adaptación.

\begin{tabular}{|c|c|c|c|c|}
\hline \multicolumn{2}{|c|}{$\mathbf{R}^{2}=\mathbf{0 , 7 1 1 7}$} & \multicolumn{2}{c|}{$\mathbf{F}^{(9,1285)}=795,9382$} & $\mathbf{0}=0,000$ \\
\hline Variables & $\mathbf{B}$ & $\hat{\mathbf{a}}$ & $\mathbf{t}$ & $\mathbf{p}$ \\
\hline Constante General & $-0,1240$ & & $-0,929$ & $\mathbf{0 , 0 0 0 0}$ \\
\hline Adaptación Escolar & 0,4529 & 0,4090 & 19,43 & $\mathbf{0 , 0 0 0 0}$ \\
\hline Adaptación Familiar & 0,3122 & 0,0949 & 4,72 & $\mathbf{0 , 0 0 0 0}$ \\
\hline Adaptación Personal & 0,2798 & $-0,0791$ & $-2,56$ & $\mathbf{0 , 0 1 0 5}$ \\
\hline
\end{tabular}




\title{
Contactar
}

Revista lberoamericana de Educación

\author{
Principal OEI
}

